\title{
Physicochemical properties of silicate-calcium micro-filler obtained by thermolysis of calcium hydrosilicates stabilized with sucrose
}

\author{
Evgeny Shoshin ${ }^{l, *}$ and Yuri Ivashchenko ${ }^{l}$ \\ ${ }^{1}$ Yuri Gagarin State Technical University of Saratov 77 Politechnicheskaya street, Saratov, Russia, \\ 410054
}

\begin{abstract}
Calcium hydrosilicates obtained by grinding a mixture of natural pozzolana (flask), $\mathrm{CaO}$ and a sucrose solution are characterized by the ability to self-disperse during heat treatment to form a highly dispersed silicate-calcium dispersion (SCD). The dependence of some physicochemical properties of the SCD (granulometric composition, occlusive capacity for sucrose) on the amount of sucrose used in the grinding of the mineral mixture is considered in the article. It was found that with an increase in the amount of sucrose used, the proportion of particles of submicron $(0.1-1 \mathrm{mkm})$ size range and the content of the residual free sucrose in the composition of the SCD increased. By varying the amount of sucrose used in the grinding of the mineral mixture, it is possible to obtain silicate-calcium mineral additives, the introduction of which into the cement paste is accompanied by the effects of accelerating or retarding the setting of the cement binder.
\end{abstract}

\section{Introduction}

The use of highly dispersed fillers is one of the main trends of cement conservation. For example, the development of self-compacting concrete technologies will allow to replace up to $70 \%$ of binders with inactive fillers, which will lead to an increase in the average world content of filler in cement to $30 \%$ by 2050 [1]. In 2013, the volume of the production of fillers for cement binders amounted to about 280 million tons per year [2] and with the development of cement replacement technologies should significantly increase. At the same time, traditional cement substitutes, such as fly ash, blast-furnace slags have a regional spread because of the insufficient volume of their production [3].Being used in the manufacture of high-strength concrete, microsilica is also a waste of the production of ferroalloys and is unevenly spread throughout the territory of the Russian Federation. At the same time, the cost of micro silica and similar pozzolanic additives (metakaolin) is several times higher than the cost of a cement binder. The use of sections to divide the text of the paper is optional and left as a decision for the author. Where the author wishes to divide the paper into sections the formatting shown in Table 2 should be used.

\footnotetext{
* Corresponding author: $\underline{\text { shoshin234@mail.ru }}$
} 
A number of mineral fillers do not have pozzolanic properties and are much cheaper than active pozzolanic additives, but in combination with effective plasticizers they provide high rheological properties of cement compositions and make it possible to reduce the consumption of the binder [4,5]. Strength of cement composites is more correlated with the water / binder ratio, where the binder is not only Portland cement and active pozzolanic additives, but also inactive fillers [1]. It means that the decrease in the amount of mixing water plays a decisive role in improving the strength properties of the composite.

A significant decrease in the amount of mixing water can be achieved by optimizing the granulometry (granulometric composition) of not only the cement but also the micrometric filler (with the particle size close to the size of the clinker particles) and the submicrometric filler (the particle size is much smaller than the clinker particle size). The combination of such mineral composition with plasticizer-dispersant additives allows to achieve $30-50 \%$ reduction in the amount of water while maintaining the given mobility of mixtures $[4,5]$. In this case, any mineral dispersion that has chemical resistance to cement stone components can be used as a filler [6,7-9].

The main source of mineral fillers in the construction industry is limestone, which is due to the availability of raw materials, relatively low energy costs for grinding [10]. A carbonate filler is not absolutely inert: during the hardening of cement, carbonate forms stabilizing ettringite and taumasite [11-14], calcium carbosilicate and carbo-aluminate hydrates $[6,11,15,16]$, which must be taken into account when choosing the parameters of the production and operation of cement products [17,18].

An alternative to carbonate fillers may be fillers based on calcium silicates which have a cement stone- related structure and do not enter into chemical reactions with its components. A related character is determined by the method of obtaining the filler, which includes the synthesis of calcium hydrosilicates under the conditions of co-grinding of silica-containing raw materials (which can be pozzolanic rocks, for example, flasks), quicklime or low-quality cement and sucrose solution [19]. Under these conditions, active formation of hydrosilicate phases is observed, the fusion of particles of which is inhibited by sucrose. Sucrose forms strong adsorption bonds with the silicate surface due to the formation of several hydrogen bonds with the siloxane groups of hydrosilicate [20], which makes sucrose a very effective stabilizing agent. The grinding of this slurry results in the accumulation of hydrosilicate phases, an important feature of which is the ability to disintegrate during heat treatment to form a dispersion containing in its composition particles of nano (less than $100 \mathrm{~nm}$ ), sub-micro (0.1-1 $\mu \mathrm{m})$ and micrometer sizes [21]. Such particle size distribution of the resulting dispersion can be useful in optimizing the multimodal particle size packing of the filler. This article discusses some of the physicochemical properties of the silicate-calcium dispersion obtained in the process of thermolysis of calcium hydrosilicates stabilized with sucrose.

\section{Materials and Methods}

\subsection{Materials}

The materials under investigation were ordinary Portland cement (OPC) of $42.5 \mathrm{H}$ type I (Holcim (rus), Russian Federation), siliceous flask extracted from the quarry in Polivanovka village of Saratov region and table sugar. The manufacture specifications are listed in Table 1, 2. 
Table 1. Characteristics of unhydrated OPC.

\begin{tabular}{|c|c|}
\hline Chemical composition (wt $\%)$ & OPC \\
\hline $\mathrm{SiO}_{2}$ & 18.7 \\
\hline $\mathrm{Al}_{2} \mathrm{O}_{3}$ & 4.6 \\
\hline $\mathrm{CaO}_{2}$ & 62.0 \\
\hline $\mathrm{Fe}_{2} \mathrm{O}_{3}$ & 3.1 \\
\hline $\mathrm{MgO}_{2}$ & 2.9 \\
\hline $\mathrm{TiO}_{2}$ & 0.3 \\
\hline $\mathrm{P}_{2} \mathrm{O}_{5}$ & 0.1 \\
\hline $\mathrm{SO}_{3}$ & 3.0 \\
\hline $\mathrm{Na}_{2} \mathrm{O}$ & 0.2 \\
\hline $\mathrm{K}_{2} \mathrm{O}$ & 0.5 \\
\hline $\mathrm{Cl}$ & 0.02 \\
\hline $\mathrm{Cl}_{3} \mathrm{~S}$ & \\
\hline $\mathrm{C}_{2} \mathrm{~S}$ & 61.1 \\
\hline $\mathrm{C}_{3} \mathrm{~A}$ & 12.6 \\
\hline $\mathrm{C}_{4} \mathrm{AF}$ & 6.8 \\
\hline $\mathrm{CaO}$ & 10.2 \\
\hline & 1.8 \\
\hline
\end{tabular}

Table 2. Characteristics of flask.

\begin{tabular}{|c|c|c|c|c|c|}
\hline \multicolumn{5}{|c|}{ Chemical composition (wt\%) } \\
\hline $\mathrm{SiO}_{2}$ & $\mathrm{Al}_{2} \mathrm{O}_{3}$ & $\mathrm{Fe}_{2} \mathrm{O}_{3}$ & $\mathrm{CaO}$ & $\mathrm{MgO}$ & п.п.п. \\
\hline 81,6 & 6,2 & $<1,5$ & 1,4 & 1,15 & 8,15 \\
\hline
\end{tabular}

\subsection{Specimen preparation}

Preparation of calcium hydrosilicates stabilized with sucrose and the products of their heat treatment was carried out by the following technique. A mixture of OPC and flask in the ratio OPC / flask $=1 / 1$ in an aqueous solution of sucrose (water / solid phase ratio of 2.0) was ground in a planetary mill at a gravitational acceleration of $\mathrm{G}=23$ for 1 hour. The sucrose content amounted to $2 \%, 5 \%, 8 \%$ by weight of the solid phase of the suspension. The mineral part of the obtained suspension was separated under reduced pressure and dried at $105{ }^{\circ} \mathrm{C}$ to constant weight. The obtained samples of calcium hydrosilicates stabilized with sucrose were subjected to heat treatment in the oven at $150{ }^{\circ} \mathrm{C}$ and at the residual pressure of $1.3-1.5 \mathrm{kPa}$ for 60 minutes.

\subsection{Experimental Procedure}

The effect of residual sucrose in the silicate-calcium dispersion (SCD) on the setting processes of modified cement pastes was evaluated on the samples of the cement paste (water / cement ratio 0,4 ) containing $30 \%$ of the SCD from the cement mass by the change in the setting time of the cement paste in accordance with GOST 310.3-76. SCDs obtained with different contents of stabilizing sucrose (2-9\%) were used for the study.

The content of residual free sugars in the composition of SCDs prepared using different amounts (2.5-16.6\% of the weight of the solid phase of the suspension) of stabilizing sucrose was determined on the samples of the corresponding aqueous extracts obtained according to GOST 21119.2-75.

The quantitative content of sucrose in the test samples of extracts was determined by the spectrophotometric method using the anthrone (9,10-Dihydro-9-oxoanthracene) reagent according to GOST (State Standard) 32080-2013 on the Evolution-300 double-beam 
spectrophotometer with the Nicolet Evolution 300 Local Control software $(\lambda=625 \mathrm{~nm}$, quartz cuvette $1=10 \mathrm{~mm}$ ).

The studies of the structure and the monitoring of the nanoparticle morphology were performed on the transmission electron microscope (TEM) CarlZeiss Libra 120, equipped with an attachment for SAED-analysis (selected area electron diffraction). Preparation of samples for the TEM was carried out according to a scheme including grinding the sample in the agate ball mill in the absolute ethanol medium followed by treatment in the ultrasonic disintegrator for $30 \mathrm{sec}$. The solid phase of the resulting suspension was separated and applied to the polymer support.

\section{Results and Discussion}

The granulometric composition of silicate-calcium dispersions (SCDs) obtained by heat treatment of calcium hydrosilicates stabilized with sucrose essentially depends on the content of stabilizing carbohydrate in the water-mineral suspension subjected to grinding, the particle size distribution curves being of a polymodal character (Fig. 3, Table 3).

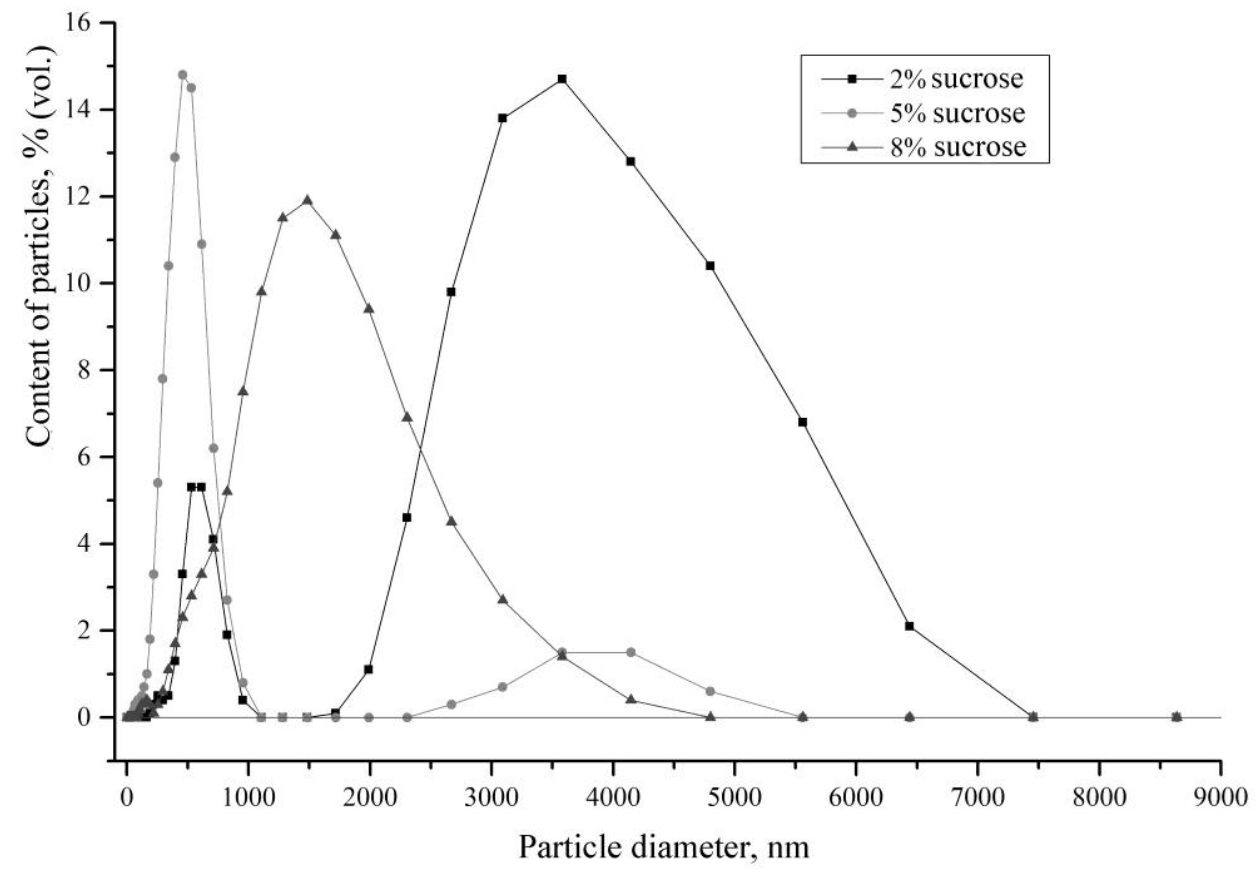

Fig. 1. Particle size distribution curves in SCD samples

Table 3. Fractional composition of SCD samples depending on the content of stabilizing sucrose

\begin{tabular}{|c|c|c|c|c|c|c|c|c|c|c|}
\hline \multirow{2}{*}{$\begin{array}{c}\text { Sucrose } \\
\text { content, } \\
\%\end{array}$} & \multicolumn{10}{|c|}{ Particle fractions, $\mu \mathrm{m}$} \\
\cline { 2 - 13 } & $<0.1$ & $\begin{array}{c}0,1- \\
0,2\end{array}$ & $\begin{array}{c}0,2- \\
0,3\end{array}$ & $\begin{array}{c}0,3- \\
0,4\end{array}$ & $\begin{array}{c}0,4- \\
0,5\end{array}$ & $0,5-1$ & $1-2$ & $2-3$ & $3-4$ & $>4$ \\
\hline 2 & 43,8 & 0,9 & 6,2 & 8,8 & 10,9 & 25,0 & 0,2 & 1,8 & 1,6 & 0,8 \\
\hline 5 & 56,9 & 19,8 & 7,8 & 7,7 & 5,1 & 2,5 & 0 & 0 & 0,1 & 0,1 \\
\hline 8 & 3,5 & 29,1 & 3,2 & 0,1 & 0,1 & 50,3 & 13,7 & 0,0 & 0,0 & 0,0 \\
\hline
\end{tabular}


The morphology of SCD particles is largely determined by their size: particles larger than $0.1 \mu \mathrm{m}$ are aggregates of irregular shape (Fig. 2a), whereas nanometer-scale particles are spherical (Fig. 2b). Nanosize particles are aggregates of smaller particles, i.e. they have a coagulative nature (Fig. 3a) and are characterized by an amorphous structure (Fig. 3b).

Taking into account that sucrose is an effective retarder of cement hardening processes, the immobilization of sucrose by a silicate matrix was further studied by the method of extraction (Table 4).

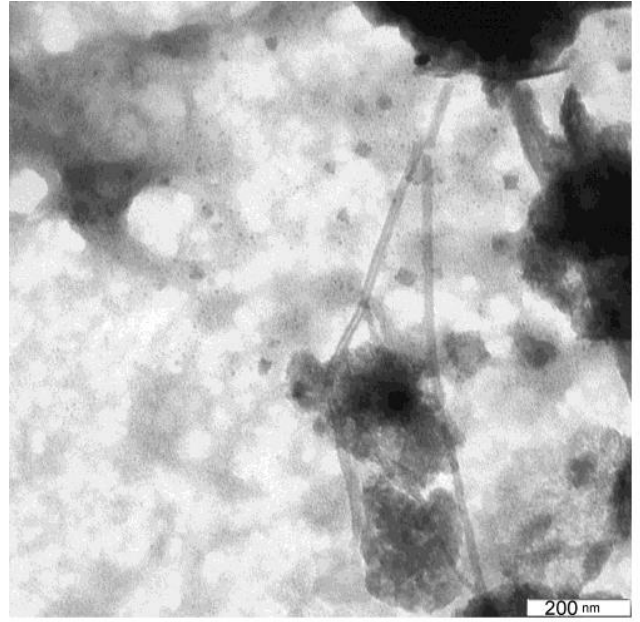

a

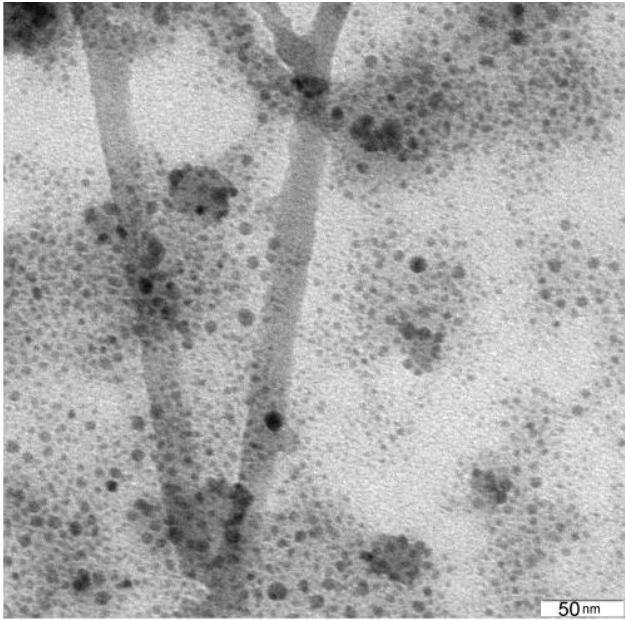

b

Fig. 2. Microelectronic photographs of SCD specimen with various magnifications

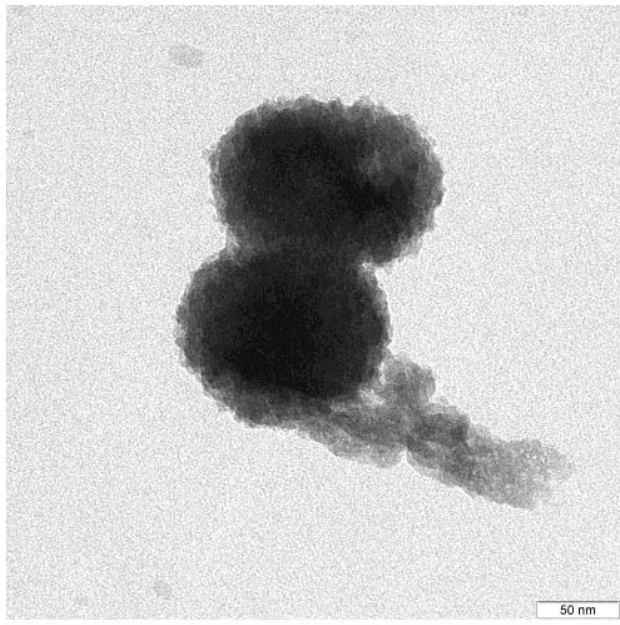

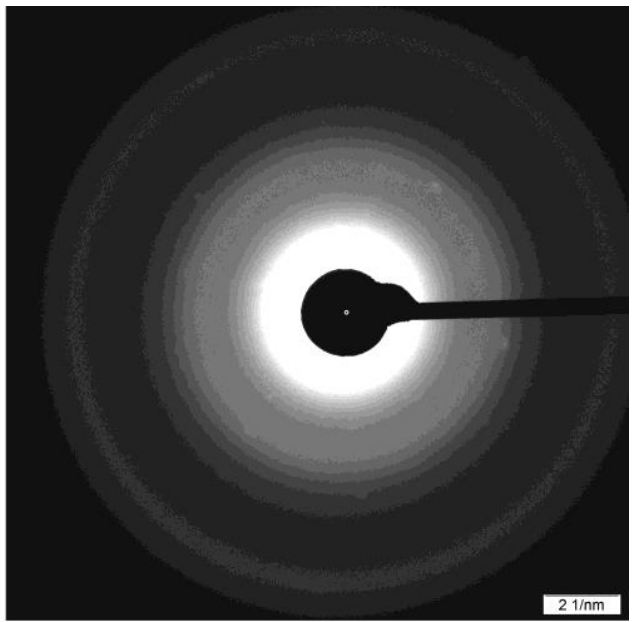

b

Fig. 3. The results of the SAED analysis of the PGM thermolysis products:

a - TEM micrograph of the aggregated particle in the composition of thermolysis products of PGM; $\mathrm{b}$ - electron diffractogram of the aggregated particle. 
Table 4. Content of residual sucrose in SCD extracts

\begin{tabular}{|c|c|c|}
\hline Initial content of sucrose in the & \multicolumn{2}{|c|}{ Content of free sucrose in the extract } \\
\cline { 2 - 3 } $\begin{array}{c}\text { composition of the water-mineral } \\
\text { suspension,\% wt. by weight of the } \\
\text { solid phase of the suspension }\end{array}$ & $\begin{array}{c}\text { Concentration of sucrose } \\
\text { in the extract, \% }\end{array}$ & $\begin{array}{c}\text { Proportion of the initial } \\
\text { amount of stabilizing } \\
\text { sucrose, } \%\end{array}$ \\
\hline 2,5 & 0,0052 & 0,372 \\
\hline 5,0 & 0,0104 & 0,374 \\
\hline 7,5 & 0,0154 & 0,369 \\
\hline 10,0 & 0,0211 & 0,381 \\
\hline 13,0 & 0,0351 & 0,486 \\
\hline 15,0 & 0,0973 & 1,167 \\
\hline 16,6 & 0,1650 & 1,789 \\
\hline
\end{tabular}

In the entire range of the used concentrations of stabilizing carbohydrate, the degree of its absorption exceeds $98 \%$ (Table 4), i.e. almost quantitative absorption of sucrose by the volume of the SCD particles is observed. However, exceeding $13 \%$ of the concentration of stabilizing sucrose leads to a sharp increase in the content of free sucrose in the extracts.

The evaluation of the effect of residual sucrose in the composition of SCD on the setting processes of cement pastes has shown that the setting processes are influenced by two effects opposite in nature: the effect of accelerating the setting in the presence of a highdispersion filler (the nucleation effect) and the effect of retarding the setting by the action of sucrose present. In the presence of SCD obtained at low concentrations of stabilizing sucrose (up to $3.5 \%$ inclusive), the nucleation effect prevails, whereas at higher concentrations of stabilizing sucrose, the effect of retardation of setting prevails (Table 5).

Table 5. Effect of the concentration of stabilizing sucrose in the composition of SCD on the setting time of modified cement pastes (SCD content is $30 \%$ of the cement weight)

\begin{tabular}{|c|c|}
\hline $\begin{array}{c}\text { Content of stabilizing sucrose in the } \\
\text { composition of SCD, } \%\end{array}$ & $\begin{array}{c}\text { Start of setting of the modified cement } \\
\text { paste, } \min (\mathrm{h})\end{array}$ \\
\hline 0 & 210 \\
2 & 105 \\
3,5 & 160 \\
5 & 370 \\
7,5 & $(9,5)$ \\
9 & $(21,0)$ \\
\hline
\end{tabular}

\section{Conclusions}

Physicochemical properties of the silicate-calcium dispersion obtained by thermolysis of calcium hydrosilicates stabilized with sucrose can be regulated in a fairly wide range by varying the concentration of stabilizing sucrose used in the preparation of calcium hydrosilicates. As the content of stabilizing sucrose increases, the fraction of particles in the range of sizes $0.1-1.0 \mu \mathrm{m}$ increases in the SCD composition, but at the same time, the content of residual free sucrose increases, which has a retarding effect on the setting processes of the cement system. It means that SCD has a multifunctional action whose character can be changed taking into account technological problems of the manufacture, which allows to consider SCD as a promising filler for cement systems. 


\section{References}

1. M. J. Vanderley, L. D. Bruno, Quattrone Marco, G. P. Rafael. Cem. and Concr. Res., (to be published)

2. CSI WBCSD, "Getting the numbers right" (GNR). Cem. Sustain. Initiat. (2013),

3. A. M. Sabbie, M. J. Vanderley, A. P. Sergio, H. Arpad. Cem. and Concr. Res. (to be published)

4. H.S. Müller, R. Breiner, J.S. Moffatt, M. Haist. Process. Eng. 95, 290 (2014)

5. T. Proske, S. Hainer, M. Rezvani, C.-A. Graubner. Cem. Concr. Res. 51, 38 (2013)

6. I. Soroka, N. Setter. Cem. Concr. Res. 7, 449 (1977)

7. W.A. Gutteridge, J.A. Dalziel. Cem. Concr. Res. 20, 778 (1990)

8. B.L. Damineli, V.M. John, B. Lagerblad, R.G. Pileggi. Cem. Concr. Res. 84, 8 (2016)

9. B.L. Damineli, F.M. Kemeid, P.S. Aguiar, V.M. John. Cem. Concr. Compos. 32, 555 (2010)

10. S. Tsivilis, E. Chaniotakis, G. Kakali, G. Batis. Cem. Concr. Compos. 24, 371 (2002)

11. B. Lothenbach, S. Le, E. Gallucci, K. Scrivener. Cem. Concr. Res. 38, 848 (2008)

12. K. De Weerdt, K.O. Kjellsen, E. Sellevold, H. Justnes. Cem. Concr. Compos. 33, 30 (2011)

13. E. Scholtzová, D. Tunega, S. Speziale. Cem. Concr. Res. 77, 9 (2015)

14. S. Köhler, D. Heinz, L. Urbonas. Cem. Concr. Res. 36, 697 (2006)

15. J. Péra, S. Husson, B. Guilhot. Cem. Concr. Compos. 21, 99 (1999)

16. J. Grandet, J.P. Ollivier. Cem. Concr. Res. 10, 759 (1980)

17. M. T. Blanco-Varela, P. M. Carmona-Quiroga, I. F. Sáez del Bosque, S. MartínezRamírez. Cem. Concr. Res. 42, 994 (2012)

18. F. Bellmann, J. Stark Cem. Concr. Res. 37, 1215 (2007)

19. E.A. Shoshin, A.V. Polyakov, A.M. Burov Bulletin BSTU. Shukhov. 3, 152 (2016)

20. B. J. Smitha, A. Rawala, G. P. Funkhouserb, L. R. Robertsc, V. Guptad, J. N. Israelachvilia, B. F. Chmelka. PNAS. 108, 8949 (2011)

21. E. A. Shoshin Building materials. 7, 16 (2017) 